\title{
Maternal Depressive Symptomatology: 16-Month Follow-up of Infant and Maternal Health-Related Quality of Life
}

\author{
Janel M. Darcy, MD, Joseph G. Grzywacz, PhD, Rebecca L. Stephens, BA, \\ Iris Leng, PhD, C. Randall Clinch, MS, DO, and Thomas A. Arcury, PhD
}

Purpose: The purpose of this study was to document risk factors for depressive symptoms during the postpartum period among working mothers and to determine longitudinal effects of depressive symptoms on maternal health-related quality of life and infant health and development.

Methods: Mother-infant dyads from a community-based cohort study of working mothers were recruited when infants were 4 months old and were interviewed every 4 months until infants were 16 months old. Depressive symptoms and health-related quality of life were assessed using the Center for Epidemiologic Studies Depression Scale and the Short Form-12 Health Survey, respectively. Infant development and health-related quality of life were measured with the Ages and Stages Questionnaire and the Infant-Toddler Quality of Life Questionnaire, respectively.

Results: Depressive symptoms were elevated among mothers who were younger, less educated, African American, unmarried, and impoverished. Mothers with significant depressive symptoms had significantly poorer physical and mental health-related quality of life, reported greater pain for their infant, and had more health-related concerns about their child. Maternal depressive symptoms at 4 months predicted infant poorer health-related quality of life at 8, 12, and 16 months.

Conclusions: Several characteristics, including age, education level, race, marital status, and poverty, can help primary care physicians identify working mothers at risk for depressive symptoms. Identification of these symptoms is important; they are correlated with poorer maternal health-related quality of life and they predict poorer children's health-related quality of life. ( $\mathrm{J}$ Am Board Fam Med 2011;24: 249-257.)

Keywords: Community Sample, Infant Development, Postpartum Depression, Quality Of Life

Postpartum depression is a common clinical and public health problem. ${ }^{1}$ Prevalence estimates from different sources suggest that $15 \%-20 \%$ of women experience one or more episodes of postpartum depression during the 12-month period after child-

This article was externally peer reviewed.

Submitted 7 September 2010; revised 10 December 2010; accepted 16 December 2010.

From the Department of Family and Community Medicine, Wake Forest University School of Medicine, Winston-Salem, NC.

Funding: This research was supported by a grant from the Eunice Kennedy Shriver National Institute for Child Health and Development (HD48601).

Conflict of interest: none declared.

Corresponding author: Joseph G. Grzywacz, PhD, Department of Family and Community Medicine, Wake Forest University School of Medicine, Medical Center Boulevard, Winston-Salem, NC 27157-1084 (E-mail: grzywacz@wfubmc.edu). birth. $^{2-4}$ Delineating the timing of onset and course of depressive symptoms during the postpartum period is elusive, in part because formal perinatal health care ends at 6 weeks postpartum. Nevertheless, evidence suggests the prevalence of major and minor postpartum depression ranges between $10 \%$ and $15 \%$ during the first 7 months of the postpartum period, and that decreases to a range of $5 \%$ to $10 \%$ for the remainder of the postpartum period. ${ }^{2}$ High prevalence across the postpartum period suggests that primary care physicians will likely encounter depression while providing care to infants or their mothers. ${ }^{5,6}$

Depressive symptoms during the postpartum period, regardless of whether they are caused by postpartum depression or a more general depressed affect, have substantial implications for women's 
health ${ }^{5,7}$ and for infants' health and development. ${ }^{6,8,9}$ Elevated depressive symptoms during the postpartum period have been associated with fewer preventive visits but greater use of acute health services for infants, including hospitalizations and emergency department visits. ${ }^{8,10}$ Similarly, elevated depressive symptoms during the postpartum period are associated with fewer developmentally enriching parenting behaviors, such as reading to the child or singing songs. ${ }^{10}$ In terms of maternal health, depression has been associated with excess weight retention after childbirth, ${ }^{11}$ compromised functional status, ${ }^{7,9}$ and somatic complaints, all of which lead to greater use of health services. ${ }^{12}$ Webb and colleagues ${ }^{7}$ also have linked depressive symptoms with a variety of maternal physical health problems such as fatigue, headache, nausea, backache, vaginal pain or dyspareunia, and urinary or bowel problems. Family physicians and other primary health care providers need to be vigilant about depression during the postpartum period given its widespread prevalence, the association with a greater consumption of health care services by depressed mothers (either for their children or themselves), and the health-related implications for mothers and their children.

Although there has been a substantial amount of research focused on depression during the postpartum period and its consequences, a major gap in the literature is the relative paucity of studies beyond the 6 -week postpartum visit. A recent comprehensive review noted that only 6 of 28 studies were designed to document depression between 4 and 12 months postpartum. ${ }^{2}$ Longitudinal studies are needed to delineate the potential consequences of depressive symptoms for maternal health later in the postpartum period. Existing longitudinal research has focused primarily on low-income mothers, ${ }^{7,8,13}$ an important group of women. However, research focusing on employed working mothers across income ranges is also needed because a large segment of mothers of infants work full-time, ${ }^{14}$ yet the postpartum experiences of these women and the implications for the subsequent well-being for those women and their children remain virtually unknown. ${ }^{3}$ Recognizing that a basic precept of family medicine is that effective treatment of an individual requires broader consideration of the family, research that examines both maternal and child outcomes is needed. Finally, additional epidemiologic research is needed to enhance under- standing of the basic distribution of depression among the population during the postpartum period. ${ }^{3}$ Such information would be invaluable to primary care physicians to help identify individuals at elevated risk for postpartum depression and its sequelae.

\section{The Present Study}

This study focuses on depressive symptoms 4 months postpartum among women who returned to full-time employment. Employed mothers of infants confront substantial strains combining work and family, strains that increase the risk of elevated depressive symptoms and psychiatric disorders. ${ }^{15,16}$ The primary aims of this study are to describe the demographics associated with screening positive for depression during the postpartum period so that family physicians may better identify mothers who are at risk for postpartum depression, and to determine the longitudinal effects of depressive symptoms 4 months postpartum on maternal and infant health-related quality of life. This study also considers the effects of depressive symptoms during the postpartum period on both the mother and child. The primary hypothesis is that depression during the postpartum period undermines subsequent health-related quality of life for both mothers and their infants.

\section{Methods}

This study is part of the "Weaving Work and Family: Implications for Mother and Child" project. The project recruited a representative community-based sample $(\mathrm{n}=217)$ of mothers of infants who returned to full-time employment (at least 30 hours per week) by the time their infants were 4 months old. Mothers were interviewed when infants were 4, 8, 12, and 16 months of age. Full details of the sampling, recruitment, and data collection protocols are available elsewhere ${ }^{17,18}$; consequently, only key points are summarized here.

No public lists are available that capture all births in the study area; therefore, the first task was to create a sample frame. The sample frame consisted of all mothers who gave birth in the primary mother-infant unit in Forsyth County, North Carolina, during a 5-month period in 2007 and who reported working during pregnancy. To be eligible for the study women needed to be 18 years of age or older and currently working at least 30 hours per 
week or planning to return to work $\geq 30$ hours per week by 4 months postpartum (when their infant was 4 months old). Women whose child had a special health care need(s) were excluded from the study. Of the 518 mothers who were selected for recruitment through a stratified, random sampling procedure to achieve a sample composition comparable to the county, 104 were unreachable because of disconnected phones or nonresponse, 10 refused to participate in eligibility screening, and 116 were ineligible, primarily because they had not returned to full-time employment by 4 months postpartum, the primarily inclusion criterion for the study. Among the 288 eligible mothers remaining, 217 (75.3\%) agreed to participate and completed a baseline interview. Of the 217 enrolled participants, 199 were seen again at the 8-month interview (91.7\%), 196 were seen again at the 12 -month interview $(90.3 \%)$, and 191 were seen again at the 16-month interview (88.0\%). A federally authorized institutional review board (FWA no. 00001435) approved all sampling, recruitment, and data collection procedures.

\section{Measures}

Depressive symptoms during the postpartum period were assessed at each interview using the Center for Epidemiologic Studies Depression Scale (CES-D). ${ }^{19}$ The CES-D is a validated instrument widely used in psychiatric epidemiology. ${ }^{20-24}$ Women who scored 16 or higher on the CES-D at the 4-month interview were defined as having significant depressive symptoms. The CES-D has been implemented in a number of other studies of postpartum depressive symptoms. ${ }^{7,8,13}$

The dependent variables in this study were maternal and infant health-related quality of life. Maternal health-related quality of life was assessed at each interview (ie, when infants were $4,8,12$, and 16 months old) using the 12-item Short Form Health Survey (SF-12). ${ }^{25}$ Published scoring algorithms were used to construct 2 composite variables reflecting mental and physical health-related quality of life (mental component score and physical component score, respectively) at each observation. Norms-based scoring based on a population mean of $50(\mathrm{SD}, 10)$ was used. Higher scores on both the mental component and physical component reflect better health-related quality of life.

Infant health-related quality of life was assessed with 3 instruments. Two dimensions of infant health-related quality of life were assessed using 2 subscales adapted from the Infant-Toddler Quality of Life instrument. ${ }^{26}$ Pain was assessed using 2 items, which asked mothers to rate the frequency and intensity of pain or discomfort experienced by the infant during the previous 4 weeks. Infant impairment was assessed with 5 items asking mothers about the extent to which their ability to attend to their own personal needs were limited by their infant's healthrelated problems (eg, feeding, sleeping, behavior) during the previous 4 weeks. Three variables reflecting developmental progression were assessed using the Ages and Stages Questionnaire: cognitive delay, motor delay, and any delay. The Ages and Stages Questionnaire is a validated, parent-administered tool that screens for delay across 5 domains of child development. ${ }^{27,28}$ Cognitive delay was defined as having a score meeting potential caseness for developmental delay in either the "language," "problem-solving," or "personal-social" subdomains of development on 2 separate observations during the 8-, $12-$, or 16-month assessments. Similarly, the motor delay variable was defined as having a score meeting potential caseness in either the "gross motor" or "fine motor" subdomains of development on 2 separate observations during the 8-, 12-, or 16month observations. The third variable was a dichotomous indicator of any delay, defined as infants whose score met potential caseness for any delay.

Infants' recent symptoms were measured using a set of common symptoms from the Warwick inventory. ${ }^{29}$ Four variables were constructed: (1) respiratory symptoms, (2) acute otitis media, (3) gastrointestinal symptoms, and (4) any recurrent illness. The first 3 system-specific indicators were defined as having at least one symptom within the illness group on 2 separate observations during the 8-, 12-, and 16 month assessments. The fourth variable was a dichotomous indicator of any recurrent illness, defined as having at least one symptom within any illness group on 2 separate observations during the 8-, 12-, and 16-month observations.

\section{Statistical Analyses}

Women with and without significant depressive symptoms were compared by basic characteristics (maternal age, race, education, marital status, and poverty status) using $\chi^{2}$ tests. Maternal and infant health-related outcomes of maternal health-related quality of life (physical and mental component sum- 
maries), infant developmental delay, recurrent infant illness, and infant health-related quality of life (pain/ discomfort and health-related concerns) were compared between mothers with and without significant depressive symptoms, using student $t$ tests for continuous variables and $\chi^{2}$ tests for categorical variables.

Two approaches were taken to assess the effects of depression during the postpartum period on infant and maternal health-related quality of life after adjusting for maternal age, race, education, marital status, and poverty. First, multiple regression models were used to assess the effects of depression on infant and maternal health-related quality of life (averaged across 8,12 , and 16 months). Then, mixed models were applied to model the longitudinal effects of depression, accounting for time, where the covariance of repeated measurements within the same subject was modeled using a compound symmetry structure. When modeling infant health-related quality of life, we also adjusted for infant birth weight and sex. In the mixed model, baseline measurements were also included as a covariate.

\section{Results}

The average age of the mothers was 30.3 years, but for analysis purposes, they were split into age groups of 18 to 24 years $(\mathrm{n}=36 ; 16.6 \%$ of the entire sample); 25 to 34 years ( $\mathrm{n}=127 ; 58.5 \%$ of the entire sample), and $\geq 35$ years $(n=54 ; 24.9 \%$ of the entire sample) (Table 1). There were 65 African-American mothers (30\% of the sample) and 152 European-American mothers (70\%). The sample included 115 mothers who had less than a college-level education (53.0\%) and 102 mothers $(47.0 \%)$ who had a college-level education or higher. The majority of mothers were married $(\mathrm{n}=$ $167 ; 77.0 \%)$ and above poverty level $(\mathrm{n}=196$; $90.3 \%)$.

Nearly one-third of the sample $(32.7 \%)$ showed significant depressive symptoms at 4 months postpartum (Table 1). Significant depressive symptoms were more common among younger women (ie, 18-24 years of age) and for African-American women compared with older women and European-American women, respectively. In addition, significant depressive symptoms were more common among those with less than a college education, those who were unmarried, and those who were living in poverty.

Bivariate comparisons indicated that symptoms of depression during the postpartum period were associated with poorer health-related quality of life for both mothers and infants. Women with significant depressive symptoms had significantly $(P=.02)$

Table 1. Baseline Demographic Characteristics by Significant Depressive Symptoms

\begin{tabular}{|c|c|c|c|c|}
\hline & Total Mothers & $\begin{array}{l}\text { Mothers with Significant } \\
\text { Depressive Symptoms* }\end{array}$ & $\begin{array}{l}\text { Mothers without Significant } \\
\text { Depressive Symptoms* }\end{array}$ & $P$ \\
\hline Total & 217 & $71(32.7)$ & $146(67.3)$ & \\
\hline Maternal age (years) & & & & .0492 \\
\hline $18-24$ & $36(16.6)$ & $18(50.0)$ & $18(50.0)$ & \\
\hline $25-34$ & $127(58.5)$ & $36(28.3)$ & $91(71.7)$ & \\
\hline $35+$ & $54(24.9)$ & $17(31.5)$ & $37(68.5)$ & \\
\hline Maternal race & & & & .0007 \\
\hline African American & $65(30.0)$ & $32(49.2)$ & $33(50.8)$ & \\
\hline European American & $152(70.0)$ & $39(25.7)$ & $113(74.3)$ & \\
\hline Maternal education & & & & .0326 \\
\hline$<$ College level & $115(53.0)$ & $45(39.1)$ & $70(60.9)$ & \\
\hline$\geq$ College level & $102(47.0)$ & $26(25.5)$ & $76(74.5)$ & \\
\hline Marital status & & & & .0030 \\
\hline Married & $167(77.0)$ & $46(27.5)$ & $121(72.5)$ & \\
\hline Unmarried & $50(23.0)$ & $25(50.0)$ & $25(50.0)$ & \\
\hline Poverty status & & & & .0027 \\
\hline At or below poverty & $21(9.7)$ & $13(61.9)$ & $8(38.1)$ & \\
\hline Above poverty & $196(90.3)$ & $58(29.6)$ & $138(70.4)$ & \\
\hline
\end{tabular}

Data provided as $\mathrm{n}(\%)$.

*Percentages refer to rows (values in rows add up to $100 \%$ ). 
poorer physical health-related quality of life scores (mean, 52.2; SD, 6.88) than did mothers without significant depressive symptoms (mean, 54.2; SD, 4.08). Women with significant depressive symptoms also had significantly $(P<.0001)$ poorer mental health-related quality of life scores (mean, 46.3; SD, 7.46) than did screen-negative mothers (mean, 52.6; $\mathrm{SD}, 4.65)$. In terms of infant health-related quality of life, significant depressive symptoms were not associated with infant developmental delay. However, a higher percentage of women with significant depressive symptoms ( $\mathrm{n}=31 ; 47.7 \%)$ reported gastrointestinal problems in their infants $(P=.0023)$ than did those without significant depressive symptoms $(\mathrm{n}=36 ; 26.1 \%)$. Furthermore, women with significant depressive symptoms self-reported greater impairment because of their child's health (mean, 1.34; $\mathrm{SD}, 0.35)$ than those without significant depressive symptoms (mean, 1.19; SD, 0.24; $P=.0041$ ).

Results from multivariate models predicting 2 indicators of infant health-related quality of life largely replicated results from bivariate analyses (Table 2). Women with significant depressive symptoms reported their children as having more pain or discomfort and reported greater impairment because of their child's (poor) health compared with women without significant depressive symptoms. Mental health-related quality of life during the later portion of the postpartum period was lower among women who were classified as having significant depressive symptoms. The bivariate association between elevated depressive symptoms and physical health-related quality of life was nonsignificant.

Longitudinal analyses controlling for time and earlier reports of infant health-related quality of life showed comparable results; infants' pain and mothers' health-related concerns at 8,12 , and 16 months were elevated among those with significant depressive symptoms (Table 3). After controlling for mental health-related quality of life earlier in the postpartum period, there was no difference in subsequent mental health-related quality of life by significant depressive symptoms status later in the postpartum period. Once baseline values of physical health-related quality of life were incorporated into the multivariate longitudinal model, trendlevel evidence $(P<.07)$ suggests that women with

Table 2. Cross-Sectional Associations of Significant Depressive Symptoms at 4-Months Postpartum with Infant and Maternal Health-Related Quality of Life*

\begin{tabular}{|c|c|c|c|c|}
\hline & \multicolumn{2}{|c|}{ Infant Health-Related Quality of Life } & \multicolumn{2}{|c|}{$\begin{array}{l}\text { Maternal Health-Related Quality of } \\
\text { Life }\end{array}$} \\
\hline & $\begin{array}{l}\text { Pain or Discomfort } \\
\quad(\mathrm{n}=186)\end{array}$ & $\begin{array}{c}\text { Mothers' Health-Related } \\
\text { Concerns } \\
(\mathrm{n}=185)\end{array}$ & $\begin{array}{c}\text { MCS } \\
(n=203)\end{array}$ & $\begin{array}{c}\text { PCS } \\
(n=203)\end{array}$ \\
\hline Depressive symptoms & $0.21(0.085)^{\dagger}$ & $0.16(0.046)^{\ddagger}$ & $-6.28(0.959)^{\ddagger}$ & $-1.26(0.825)$ \\
\hline \multicolumn{5}{|l|}{ Covariates } \\
\hline \multicolumn{5}{|l|}{ Age (years) } \\
\hline $18-24$ & $-0.16(0.154)$ & $-0.15(0.083)$ & $-1.76(1.703)$ & $2.05(1.465)$ \\
\hline $25-34$ & $0.07(0.088)$ & $-0.03(0.048)$ & $0.46(1.006)$ & $1.66(0.866)$ \\
\hline \multicolumn{5}{|l|}{ Maternal race } \\
\hline African American & $-0.29(0.105) \S$ & $-0.11(0.057)$ & $0.21(1.162)$ & $-0.29(1.000)$ \\
\hline \multicolumn{5}{|l|}{ Maternal education } \\
\hline$\geq$ College & $-0.10(0.083)$ & $-0.07(0.045)$ & $0.67(0.956)$ & $0.66(0.823)$ \\
\hline \multicolumn{5}{|l|}{ Marital status } \\
\hline Married & $0.19(0.124)$ & $0.02(0.067)$ & $-0.86(1.455)$ & $4.05(1.252) \S$ \\
\hline \multicolumn{5}{|l|}{ Poverty status } \\
\hline At/below poverty & $0.24(0.182)$ & $0.01(0.098)$ & $-0.35(2.035)$ & $1.40(1.751)$ \\
\hline
\end{tabular}

All data provided as $\beta$ (SE).

${ }^{*}$ Reference groups in the models are women aged 35+, European American, less than college education, unmarried, and above poverty. Models for children also control for child sex and birth weight.

${ }^{\dagger} P \leq .05$.

${ }^{\ddagger} P \leq .001$.

${ }^{\S} P \leq .01$.

MCS, Mental component summary from the 12-item Short Form; PCS, physical component summary from the 12-item Short Form. 
Table 3. Longitudinal Effects of Significant Depressive Symptoms at 4 Months Postpartum on Infant and Maternal Health-Related Quality of Life at 8, 12, and 16 Months Postpartum (Adjusted)*

\begin{tabular}{|c|c|c|c|c|}
\hline & \multicolumn{2}{|c|}{ Infant Health-Related Quality of Life } & \multicolumn{2}{|c|}{$\begin{array}{c}\text { Maternal Health-Related Quality of } \\
\text { Life }\end{array}$} \\
\hline & $\begin{array}{l}\text { Pain/Discomfort } \\
\quad(\mathrm{n}=203)\end{array}$ & $\begin{array}{l}\text { Health-Related Concerns } \\
\qquad(\mathrm{n}=203)\end{array}$ & $\begin{array}{c}\text { MCS } \\
(\mathrm{n}=202)\end{array}$ & $\begin{array}{c}\text { PCS } \\
(\mathrm{n}=202)\end{array}$ \\
\hline Depressive symptoms & $0.24(0.080)^{\dagger}$ & $0.14(0.042)^{\dagger}$ & $-1.20(0.045)$ & $-1.19(0.699)$ \\
\hline \multicolumn{5}{|l|}{ Covariates } \\
\hline \multicolumn{5}{|l|}{ Age (years) } \\
\hline $18-24$ & $-0.09(0.141)$ & $-0.10(0.075)$ & $-1.53(1.415)$ & $1.11(1.233)$ \\
\hline $25-34$ & $0.08(0.085)$ & $-0.01(0.045)$ & $0.22(0.856)$ & $0.69(0.748)$ \\
\hline \multicolumn{5}{|l|}{ Maternal race } \\
\hline African American & $-0.26(0.101)^{\ddagger}$ & $-0.12(0.053)^{\ddagger}$ & $-0.51(0.975)$ & $0.29(0.850)$ \\
\hline \multicolumn{5}{|l|}{ Maternal education } \\
\hline$\geq$ College & $0.00(0.080)$ & $-0.06(0.042)$ & $0.88(0.802)$ & $0.06(0.702)$ \\
\hline \multicolumn{5}{|l|}{ Marital status } \\
\hline Married & $0.13(0.114)$ & $0.02(0.060)$ & $-0.85(1.162)$ & $3.22(1.007)^{\dagger}$ \\
\hline \multicolumn{5}{|l|}{ Poverty status } \\
\hline At/below poverty & $0.14(0.164)$ & $-0.02(0.086)$ & $-1.00(1.672)$ & $2.80(1.441)$ \\
\hline Baseline & $0.22(0.056)^{\S}$ & $0.25(0.050)^{\S}$ & $0.51(0.057)^{\S}$ & $0.33(0.053)^{\S}$ \\
\hline
\end{tabular}

All data provided as $\beta$ (SE).

* Reference groups in the models are women aged $35+$ years, European American, less than college education, unmarried, and above poverty. All models control for the effects of time and earlier assessments of health-related quality of life. Models for children also control for child sex and birth weight.

${ }^{\dagger} P \leq .01$.

${ }^{\ddagger} P \leq .05$.

${ }^{\S} \leq \leq .001$.

MCS, Mental component summary from the 12-item Short Form; PCS, physical component summary from the 12-item Short Form.

significant depressive symptoms may have lower physical health-related quality of life at 8,12 , and 16 months postpartum.

\section{Discussion}

Depression and other psychiatric disorders during the postpartum period have significant clinical implications for women and their infants. ${ }^{1}$ Although existing primary care and epidemiologic research offers information needed for enhanced referral and care, research that assesses depressive symptoms throughout the postpartum period and evaluating subsequent health implications for both women and their children is needed. ${ }^{2,3}$ This study was designed to assist family physicians and other primary care providers in identifying women at risk for postpartum depression and to evaluate the potential role that postpartum depression may play in subsequent clinical encounters with employed mothers or their children.

Nearly one-third of this sample of employed mothers (32.7\%) reported significant depressive symptoms at 4 months postpartum. This estimate is higher than the $10 \%$ to $15 \%$ point prevalence estimates reported in a previous review of the postpartum depression literature ${ }^{2}$ and the $15 \%$ prevalence rate reported from a nationally representative sample. ${ }^{4}$ The heightened rate of significant depressive symptoms in this study could reflect 3 possibilities. First, the elevated rate could reflect the added strain of combining full-time work and family and the subsequent elevated risk for depression experienced by working mothers. ${ }^{30,31}$ Second, recognizing that mood and anxiety symptoms frequently occur together, ${ }^{32,33}$ it is possible that the CES-D was picking up on symptoms of both disorders. Consistent with this possibility, Vesga-Lopez and colleagues ${ }^{4}$ reported that $27.5 \%$ of postpartum women reported any mood or anxiety disorder. Third, it is possible the CES-D produced too many false positives, perhaps because of elevated somatic symptoms. ${ }^{34}$ Although future research will need to explore each explanation, the bottom line remains the same: a substantial proportion of working mothers of infants 
experience elevated mental health symptoms several months beyond the typical observation period for postpartum depression.

Being classified as having significant depressive symptoms was not equally distributed among our sample. Mothers who were younger, African American, had less than a college degree, were unmarried, and/or were living in poverty were more likely to show significant depressive symptomatology. These results, which are consistent with previous research not focused on working mothers of infants, ${ }^{4,13,35}$ suggest that some women are more vulnerable to postpartum depression, perhaps because of insufficient preparation for motherhood or because of insufficient social or financial supports to help shoulder the task of working full-time while caring for an infant. ${ }^{36}$ It is important that health care providers remain cognizant of these factors so they can more accurately identify women at risk for postpartum depression. Early identification and referral are important to minimize morbidity. ${ }^{5}$ Monitoring potential depression among women who return to work provides a strong opportunity for patient-centered care because evidence suggests that mothers would like their prenatal and postpartum health care providers to discuss issues surrounding return to work after childbirth. ${ }^{17}$

The primary contribution of this study is the observed associations between depressive symptoms at 4 months postpartum and subsequent infant and maternal health-related quality of life. Bivariate results showed a higher incidence of gastrointestinal symptoms among children of mothers with significant depressive symptoms than among children of mothers without significant symptoms. These results are similar to effects described by MosesKolko and Roth ${ }^{37}$ and they are congruent with research by others, ${ }^{38}$ indicating that maternal postpartum depression predicted higher incidence of infant diarrhea. Our multivariate longitudinal analyses also indicated robust associations between significant maternal depressive symptoms and 2 domains of infant health-related quality of life: infant pain or discomfort and the extent to which infant health concerned the mother. These results are consistent with previous research indicating that postpartum depression was associated with poorer infant health and development. ${ }^{6,8,9}$ Given the nature of these self-report data, it is not clear if infants of mothers with significant depressive symptoms were, in fact, experiencing more pain and discom- fort, or whether these mothers somaticized their infants' behavior. Regardless, primary health care providers need to be attentive to maternal depression because it will likely result in greater infant health visits either because the infant is genuinely sick or because the mother believes the child is sick. Indeed, previous evidence suggests that postpartum maternal depression is associated with greater use of health services by infants. ${ }^{8,10}$

Analyses revealed little evidence that elevated depressive symptoms during the postpartum period had lasting implications for working mothers' physical and mental health-related quality of life. Null effects were surprising in light of results from previous research that suggested that postpartum depression predicted excess weight retention, ${ }^{11}$ poorer functional status, ${ }^{7,9}$ and greater somatic complaints. ${ }^{12}$ Nevertheless, it is possible that the effects of elevated depressive symptoms on health-related quality of life is attenuated for women who select to return to the full-time labor force, ${ }^{39}$ or that the sheer demands of working fulltime while mothering an infant allows little opportunity for compromised role performance or other domains of health-related quality of life assessed by SF-12. Although replication research and subsequent explorations of possible explanations are needed, these results suggest that significant depressive symptoms may be less debilitating among working mothers than among women in general.

A primary strength of this study is the longitudinal data, which allowed us to analyze the effects of maternal postpartum depressive symptoms over an extended period of time, providing a unique contribution to the postpartum depression literature. The vast majority of previous longitudinal research on postpartum depression only covers the first few months postpartum, with most only considering the first 6 weeks of postnatal care. This project, on the other hand, extended 16 months after the infants were born, providing a glimpse at the impact of maternal depression even further into the infant's life. Furthermore, we analyzed both mother and child health outcomes in relation to maternal depressive symptoms during the postpartum period. There is a lack of previous research that looks at effects on both the mother and child.

Nevertheless, the limitations of this study need to be acknowledged. An important limitation is reliance on single sources of data. Although assessment of infant health and well-being ultimately 
rests on proxy report, heavy reliance on parental report raises potential response biases. Future research using stronger, more clinically endorsed assessments would contribute to this body of research. A second limitation is that our sample only included European and African Americans. There were no other races or ethnicities included in the sample frame, so we do not have information to determine whether other groups of mothers would be more or less likely to screen positive for postpartum depression. Results tell us that AfricanAmerican mothers may be more susceptible to postpartum depression, but we cannot be sure whether or not this trend is similar for other minorities. A third limitation is that there were 104 mothers in the sample frame who could not be contacted and thus were not able to participate, which may have produced some selection biases.

\section{Conclusion}

Limitations notwithstanding, the cross-sectional results of this study suggest that postpartum depression is associated with poorer infant and maternal health-related quality of life. The data clearly suggest that that a large proportion of working mothers experience elevated symptoms of depression, and our longitudinal results show that that significant depressive symptoms during the postpartum period predicts subsequent poorer infant health-related quality of life. Even though further research is needed to determine if the observed relationship is causal, it is likely that lower levels of infant health-related quality of life will elicit additional seeking of health care for the child. Consistent with the a basic precept of family medicine that effective treatment of an individual requires broader consideration of the family, it is important that health care providers screen for and consider the role of postpartum depression when diagnosing and creating a treatment plan for the child.

\section{References}

1. Wisner KL, Chambers C, Sit DKY. Postpartum depression: a major public health problem. JAMA 2006;296:2616-8.

2. Gaynes BN, Gavin N, Meltzer-Brody S, et al. Perinatal depression: prevalence, screening, accuracy, and screening outcomes. Evid Rep Technol Assess (Summ) 2005;(119):1-8.

3. O'Hara MW. Postpartum depression: what we know. J Clin Psychology 2009;65:1258-69.
4. Vesga-Lopez O, Blanco C, Keyes K, et al. Psychiatric disorders in pregnant and postpartum women in the United States. Arch Gen Psychiatry 2008;65:805-15.

5. Berg AO. Screening for depression: recommendations and rationale. Am Fam Physician 2002;66:647-50.

6. Gjerdingen DK, Yawn BP. Postpartum depression screening: importance, methods, barriers, and recommendations for practice. J Am Board Fam Med 2007;20:280-8.

7. Webb DA, Bloch JR, Coyne JC, et al. Postpartum physical symptoms in new mothers: their relationship to functional limitations and emotional wellbeing. Birth 2008;35:179-87.

8. Chung EK, McCollum KF, Elo IT, et al. Maternal depressive symptoms and infant health practices among low-income women. Pediatrics 2004;113:e523-9.

9. Posmontier B. Functional status outcomes in mothers with and without postpartum depression. J Midwifery Womens Health 2008;53:310-8.

10. Minkovitz CS, Strobino D, Scharfstein D, et al. Maternal depressive symptoms and children's receipt of health care in the first 3 years of life. Pediatrics 2005;115:306-14.

11. Herring SJ, Oken E, Haines J, et al. Misperceived pre-pregnancy body weight status predicts excessive gestational weight gain: findings from a US cohort study. BMC Pregnancy Childbirth 2008;8:54.

12. Gold LH. Postpartum disorders in primary care: diagnosis and treatment. Prim Care 2002;29:27-41.

13. Mora PA, Bennett IM, Elo IT, et al. Distinct trajectories of perinatal depressive symptomatology: evidence from growth mixture modeling. Am J Epidemiol 2009;169:24-32.

14. US Department of Labor, Bureau of Labor Statistics. News. Employment characteristics of families in 2008. Available at: http://www.bls.gov/news.release/ archives/famee_05272009.pdf. Accessed 13 March 2011.

15. Frone MR. Work-family conflict and employee psychiatric disorders: The national comorbidity survey. J App Psychol 2000;85:888-95.

16. Killian T, Turner J, Cain R. Depressive symptoms of caregiving women in midlife: The role of physical health. J Women Aging 2005;17:115-27.

17. Clinch CR, Grzywacz JG, Tucker J, et al. Characteristics of mother-provider interactions surrounding postpartum return to work. J Am Board Fam Med 2009;22:498-506.

18. Grzywacz JG, Tucker J, Clinch CR, et al. Individual and job-related variation in infant feeding practices among working mothers. Am J Health Behav 2010; 34:186-96.

19. Radloff LS. The CES-D scale: a self-report depression scale for research in the general population. App Psychol Meas 1977;1:385-401.

20. Hales DP, Dishman RK, Motl RW, et al. Factorial validity and invariance of the center for epidemiologic 
studies depression (CES-D) scale in a sample of black and white adolescent girls. Ethn Dis 2006;16:1-8.

21. Knight RG, Williams S, Mcgee R, et al. Psychometric properties of the Centre for Epidemiologic Studies Depression Scale (CES-D) in a sample of women in middle life. Behav Res Ther 1997;35:373-80.

22. Orme JG, Reis J, Herz EJ. Factorial and discriminant validity of the Center for Epidemiologic Studies Depression (Ces-D) scale. J Clin Psychol 1986;42: 28-33.

23. Pickard AS, Dalal MR, Bushnell DM. A comparison of depressive symptoms in stroke and primary care: applying Rasch models to evaluate the Center for Epidemiologic Studies-Depression Scale. Value Health 2006;9:59-64.

24. Stahl D, Sum CF, Lum SS, et al. Screening for depressive symptoms: validation of the Center for Epidemiologic Studies Depression Scale (CES-D) in a multiethnic group of patients with diabetes in Singapore. Diabetes Care 2008;31:1118-9.

25. Ware JE, Kosinski M, Keller SD. A 12-item shortform health survey: construction of scales and preliminary tests of reliability and validity. Med Care 1996;34:220-33.

26. Landgraf JM. The infant/toddler quality of life questionnaire: conceptual framework, logic, content and preliminary psychometric results. Final Report to Schering-Plough Laboratories and Health Technology Associates. Boston: New England Medical Center; 1994.

27. Bricker D, Squires J, Mounts L. Ages and Stages questionnaire: a parent-completed, child-monitoring system. Baltimore, MD: Brookes, 1995.

28. Squires J, Bricker D, Potter L. Revision of a parentcompleted developmental screening tool: Ages and Stages questionnaires. J Pediatr Psychol 1997;22:313-28.

29. Spencer NJ, Coe C. The development and validation of a measure of parent-reported child health and morbidity: the Warwick Child Health and Morbidity Profile. Child Care Health Dev 1996;22:367-79.

30. Frone MR, Yardley JK. Workplace family-supportive programmes: Predictors of employed parents' importance ratings. J Occup Organ Psychol 1996;69: 351-66.

31. Grzywacz JG, Bass BL. Work, family, and mental health: testing different models of work-family fit. J Marriage Fam 2003;65:248-61.

32. Costa PT, McCrae RR. Revised NEO Personality Inventory and NEO Five-Factor Inventory Professional Manual. Odessa, FL: Psychological Assessment Resources; 1992.

33. Watson D, Clark LA, Weber K, et al. Testing a tripartite model. 2. Exploring the symptom structure of anxiety and depression in student, adult, and patient samples. J Abnorm Psychol 1995;104:15-25.

34. Sharp LK, Lipsky MS. Screening for depression across the lifespan: a review of measures for use in primary care settings. Am Fam Phys 2002;66:1001-8.

35. Howell EA, Mora PA, Horowitz CR, et al. Racial and ethnic differences in factors associated with early postpartum depressive symptoms. Obstet Gynecol 2005;105:1442-50.

36. Tucker J, Grzywacz JG, Leng I, et al. Return to work, economic hardship, and women's postpartum health. Women Health 2010;50:618-38.

37. Moses-Kolko EL, Roth EK. Antepartum and postpartum depression: healthy mom, healthy baby. J Am Med Womens Assoc 2004;59:181-91.

38. Rahman A, Iqbal Z, Bunn J, et al. Impact of maternal depression on infant nutritional status and illness: a cohort study. Arch Gen Psychiatry 2004;61:946-52.

39. Repetti RL, Matthews KA, Waldron I. Employment and womens health: effects of paid employment on womens mental and physical health. Am Psychol 1989;44:1394-401. 